\title{
Enredos na trajetória percorrida por mulheres até o tratamento quimioterápico
}

\author{
Tangle in the trajectory taken by women \\ until the chemotherapy treatment
}

\section{Tais da Silva Neujahr ${ }^{1}$ Adrize Rutz Porto² Kamila Dias Gonçalves 3 (1) Maira Buss Thofehrn 4 (1) Helena Ribeiro Hammes ${ }^{5}$ (C) Aline Koehler Geppert ${ }^{6}$ (C)}

\begin{abstract}
1'Autora para correspondência. Universidade Federal de Pelotas (Pelotas). Rio Grande do Sul, Brasil. taisneujahr1@hotmail.com 2-6Universidade Federal de Pelotas (Pelotas). Rio Grande do Sul, Brasil. adrizeporto@gmail.com, kamila_goncalves_@hotmail.com, mairabusst@hotmail.com, helenahammes@yahoo.com.br, aline.geppert@hotmail.com
\end{abstract}

RESUMO | OBJETIVO: Conhecer a trajetória percorrida pelas mulheres desde os primeiros sintomas do câncer ao tratamento quimioterápico na rede do Sistema Único de Saúde em um município no sul do Brasil. MÉTODO: estudo qualitativo, descritivo, realizado em unidade de tratamento quimioterápico de um hospital universitário federal, em que 12 mulheres, com qualquer tipo de câncer, foram selecionadas por conveniência e entrevistadas, em outubro de 2015. Os dados foram tratados por análise de conteúdo. RESULTADOS: A partir dos relatos das entrevistadas, notou-se que as trajetórias não foram de forma retilínea, nem convergiam em um fluxo definido de atenção às pessoas com câncer na rede de saúde pesquisada, de modo que algumas mulheres tiveram a primeira consulta com médico especialista. 0 acesso às consultas e aos exames para diagnóstico do câncer foi demorado no sistema público de saúde, mas o tratamento, foi em menor tempo. CONSIDERAÇÕES FINAIS: O investimento na elaboração de um fluxo específico de atenção às pessoas com câncer na rede de saúde e na capacitação dos profissionais da saúde, quanto a isso e sobre estratégias de orientação aos usuários do sistema pode otimizar a detecção precoce e proporcionar maiores chances de sobrevida e cura para as pacientes.

PALAVRAS-CHAVE: Enfermagem. Acesso aos serviços de saúde. Assistência integral à saúde. Saúde da mulher.
ABSTRACT | OBJECTIVE: To know the trajectory taken by women as of the first symptoms of cancer up to the chemotherapy treatment in the Brazilian Unified Health System in a city in the south of Brazil. METHOD: A qualitative, descriptive study was conducted in the chemotherapy unit of a federal teaching hospital, in which 12 women, with any type of cancer, were selected by convenience and interviewed in October 2015. Data were submitted to content analysis. RESULTS: The statements of the women interviewed showed that their paths were not horizontal, nor converged to a defined care flow for people with cancer in the studied network, and some of them had their first appointment with a specialist physician. Access to appointments and cancer diagnosis was delayed in the public health system, but treatment was faster. FINAL CONSIDERATIONS: Investment in the development of a specific care flow for people with cancer in the healthcare network and in the qualification of health professionals regarding this care and guidance strategies for system users could optimize early detection and provide patients with greater chances of survival and cure.

KEYWORDS: Nursing. Health services accessibility. Comprehensive health care. Women's health. 


\section{Introdução}

A detecção precoce do câncer melhora o prognóstico, pois quanto mais rápido o diagnóstico, maiores são as chances de sobrevida e cura do câncer. A trajetória percorrida pelas pacientes vai desde o surgimento dos primeiros sinais e sintomas, até a definição do diagnóstico. Esse processo até o início do tratamento de uma doença requer agilidade e resolubilidade, em especial para aquelas pessoas diagnosticadas com câncer ${ }^{1}$.

Dessa forma, entender o fluxo que a paciente percorre na rede do Sistema Único de Saúde (SUS), desde os primeiros sintomas até o tratamento, instiga o estudo das linhas de cuidado em atenção oncológica. Essas ações visam a redução da morbidade e a melhor utilização dos recursos disponíveis em cada nível de atenção ${ }^{2}$. Assim, é previsto: consulta com generalista para rastreamento e diagnóstico precoce, consulta especializada visando a investigação, diagnóstico com exames e biópsia, tratamento cirúrgico, quimioterápico, radioterápico e hormonioterapia ou cuidados paliativos. A atenção integral aparenta fácil acesso da primeira consulta até o tratamento, entretanto, barreiras fazem parte desse caminho.

Estudo aponta ruídos de comunicação tanto de caráter equivocado, quanto insuficiente dos serviços da rede de saúde, bem como informações incompletas, com baixa resolutividade nas condutas e esclarecimento das etapas até o tratamento3 que culminam no agravamento do câncer e aparecimento de metástases, também decorrentes do tempo de espera pelos exames diagnósticos e para o início do tratamento, em que a usuária associa o serviço privado com caráter complementar ao serviço público.4 Nesse sentido, é ressaltado o despreparo dos profissionais para investigar a doença e encaminhar aos serviços da rede de saúde ${ }^{5}$, de modo que suas orientações e condutas são bastante significativas no percurso da pessoa com câncer na rede de saúde ${ }^{6}$.
Historicamente, o cuidado com a saúde ainda é visto, na divisão clássica dos papéis entre os gêneros, como uma função mais associada às mulheres, isso porque, existem masculinidades hegemônicas, isto é, o forte vínculo entre saúde e a responsabilidade de provedor da família. Problemas de saúde, para os homens, estariam possivelmente relacionados ao fato de prejudicar a família, uma vez que comprometeria a função do homem no sustento do lar, tendo em vista o seu lugar culturalmente atribuído enquanto sujeito do trabalho e do lar $^{7}$. A mortalidade por câncer de homens é de 115 mil e de mulheres 104 mil em $2017^{8}$.

Estudos realizados em municípios dos estados do Mato Grosso, Rio de Janeiro, Paraná e Rio Grande do Sul apontam que o itinerário diagnóstico-terapêutico são caracterizados por fragilidades de articulação da rede de atenção, dificuldades na oferta e planejamento dos serviços na atenção ao paciente oncológico ${ }^{3-4,6,9}$. Há uma lacuna de conhecimento no que tange prover o percurso mais rápido às pessoas, independentemente do seu câncer, de modo a priorizar o acesso ao diagnóstico precoce, em qualquer ponto da rede de atenção, do sistema público de saúde. Pesquisas sobre o tema podem contribuir para a elaboração de estratégias eficazes, que visem melhorar a atenção de saúde das pessoas, com possíveis sintomas de câncer.

Diante dos caminhos trilhados pelas pessoas para acessar a rede de saúde em busca de cuidados aos seus sintomas, diagnóstico e tratamento, objetivou-se conhecer a trajetória percorrida pelas mulheres desde os primeiros sintomas do câncer ao tratamento quimioterápico do SUS em um município no sul do Brasil. 


\section{Método}

Trata-se de um estudo qualitativo, do tipo descritivo, realizado em uma unidade de tratamento quimioterápico de um hospital universitário federal, que atende integralmente pelo SUS. Os critérios de inclusão de participantes no estudo foram: ser mulher, ter câncer, estar em tratamento quimioterápico, estar comunicativa, orientada e lúcida, ter mais de 18 anos de idade. A escolha por participantes do sexo feminino, neste estudo, foi por buscarem mais frequentemente os serviços de saúde, do que os homens e em tratamento quimioterápico, enquanto ponto final da trajetória, para abarcar diversidade de estágios da doença nas participantes do estudo e contribuir para os diferentes trajetos percorridos ao longo da jornada até o tratamento na rede SUS.

Além disso, a seleção dos participantes do estudo se deu por conveniência, convidando-se àquelas mulheres que se encontravam em tratamento quimioterápico, por ocasião da coleta de dados e o fechamento do número de participantes da pesquisa se deu por saturação teórica, não se incluindo mais participantes, quando se verificou certa redundância nos dados obtidos na pesquisa. Assim, 12 mulheres participaram da pesquisa.

A coleta de dados, por meio de entrevista semiestruturada, foi realizada, no mês de outubro de 2015, por uma acadêmica de enfermagem, previamente capacitada pela orientadora da pesquisa, com titulação de doutorado em enfermagem. A entrevista foi guiada por roteiro, contendo questões sobre: primeiros sintomas da doença; descoberta da doença; a busca do diagnóstico da doença; região do corpo que a doença afetou e como foi para conseguir o tratamento de quimioterapia.

As entrevistas ocorreram no próprio local de estudo, de forma individual, com data e hora pré-estabeleci$\mathrm{da}$, conforme contato com as participantes, durante a realização da quimioterapia, e aceitação em participar do estudo. Todas as mulheres convidadas, aceitaram participar da pesquisa. As entrevistas foram gravadas em áudio, tendo duração, em média, de 30 minutos, sendo posteriormente, transcritas.
Os preceitos éticos foram respeitados, a pesquisa norteou-se pela Resolução n. ${ }^{\circ} 466 / 2012$ do Conselho Nacional de Saúde que trata da pesquisa com seres humanos e o projeto foi aprovado, sob o número de certificado de apresentação para apreciação ética 49217115500005316 e de parecer 1248555 . As participantes foram identificadas por meio de um codinome, precedidos da letra $\mathrm{M}$ de mulher e números arábicos (M1, M2, M3...).

Os dados foram tratados por meio da análise de conteúdo.10 Iniciou-se pela pré-análise, lendo-se os trechos transcritos da entrevista e organizando-se o material. Posteriormente, o material foi explorado por meio de leitura exaustiva, destacando-se os tópicos mais relevantes de acordo com o objetivo do estudo e agrupando-se em temas e, por fim, a análise final, comparou-se os achados, estabelecendo relações com a literatura científica disponível.10 Os temas foram: primeiras manifestações do câncer; trajetória dos primeiros sintomas até o diagnóstico e trajetória do diagnóstico de câncer até acesso ao tratamento quimioterápico.

\section{Resultados}

As principais características das participantes do estudo são: idade variou de 35 a 69 anos, com média de 55 anos, a escolaridade variou do ensino fundamental incompleto a ensino superior incompleto, apenas três delas nunca fumaram, quatro somente referiram história de câncer na família, cinco estavam em tratamento do câncer de mama, duas com câncer na bexiga e outras no pulmão, estômago, útero e vulva e ainda uma tinha mieloma múltiplo.

As mulheres relataram o caminho que percorreram para acessar o tratamento quimioterápico, desde o início dos sintomas/primeiras manifestações do câncer e, por meio de seus depoimentos foi possível elaborar o Quadro 1, dessa trajetória percorrida. Todas, no momento da coleta de dados, em outubro de 2015, encontravam-se em tratamento quimioterápico endovenoso. 


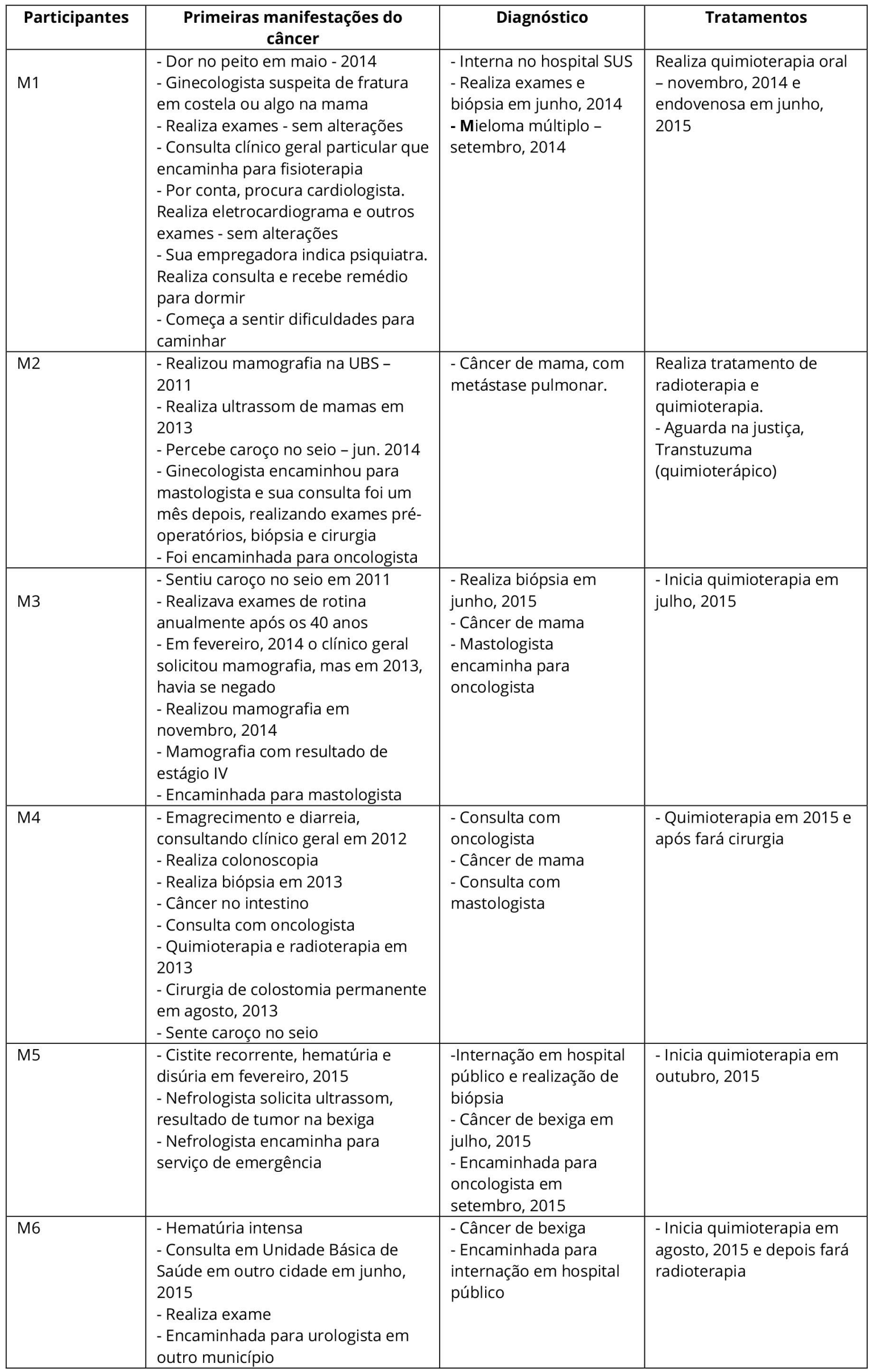




\begin{tabular}{|c|c|c|c|}
\hline Participantes & $\begin{array}{l}\text { Primeiras manifestações do } \\
\text { câncer }\end{array}$ & Diagnóstico & Tratamentos \\
\hline M7 & $\begin{array}{l}\text { - Caroço no seio, } 2010 \\
\text { - Na Unidade Básica consultou com } \\
\text { enfermeiro e realizou consulta de } \\
\text { exame citopatológico, sendo } \\
\text { solicitada mamografia } \\
\text { - Consultou com clínico geral da } \\
\text { Unidade } \\
\text { - Encaminhamento para } \\
\text { mastologista }\end{array}$ & $\begin{array}{l}\text { - Realizou biópsia } \\
\text { - Câncer de mama }\end{array}$ & $\begin{array}{l}\text { - Mastectomia radical a } \\
\text { direita } \\
\text { - Encaminhamento para } \\
\text { oncologista } \\
\text { - Radioterapia em } \\
\text { dezembro, } 2010 \\
\text { - Quimioterapia oral em } \\
\text { setembro, } 2011 \\
\text { - Quimioterapia, } 2015 \text {. }\end{array}$ \\
\hline M8 & $\begin{array}{l}\text { - Nódulo no seio em dezembro, } 2009 \\
\text { - Realiza mamografia e ultrassom } \\
\text { - Consulta clínico geral particular e } \\
\text { realiza exames } \\
\text { - Mastologista pelo sistema público } \\
\text { de saúde }\end{array}$ & $\begin{array}{l}\text { - Biópsia em 18/janeiro, } \\
2010 \\
\text { - Câncer de mama }\end{array}$ & $\begin{array}{l}\text { - Cirurgia por agulhamento } \\
\text { em 25/janeiro, } 2010 \\
\text { - Em fevereiro, oncologista } \\
\text { pelo sistema público de } \\
\text { saúde } \\
\text { - Quimioterapia } \\
\text { endovenosa e } \\
\text { radioterapia, } 2010 \\
\text { - Quimioterapia oral em } \\
\text { dezembro, } 2010 \\
\text { - Quimioterapia, } 2015\end{array}$ \\
\hline M9 & $\begin{array}{l}\text { - Dor no estômago } \\
\text { - Consulta com clínico geral de } \\
\text { convênio, } 2014\end{array}$ & $\begin{array}{l}\text { - Solicitação de } \\
\text { endoscopia e biópsia } \\
\text { - Câncer de estômago } \\
\text { - Encaminhada para } \\
\text { cirurgião } \\
\text { - Cirurgia bariátrica e } \\
\text { encaminhamento para } \\
\text { oncologista }\end{array}$ & $\begin{array}{l}\text { - Inicia quimioterapia em } \\
\text { junho, } 2014 \\
\text { - Perdeu convênio } \\
\text { - Encaminhada pelo } \\
\text { sistema público para } \\
\text { oncologista } \\
\text { - Tomografia, resultado } \\
\text { tumor de abdômen } \\
\text { - Inicia quimioterapia } \\
\text { setembro, } 2015\end{array}$ \\
\hline M10 & $\begin{array}{l}\text { - Exame de rotina na Unidade Básica } \\
\text { - Alteração em raio-X de pulmão } \\
\text { - Encaminhada para internação } \\
\text { hospitalar }\end{array}$ & $\begin{array}{l}\text { - Realiza biópsia } \\
\text { - Câncer de pulmão } \\
\text { - Encaminhada para } \\
\text { oncologista }\end{array}$ & $\begin{array}{l}\text { - Inicia radioterapia e } \\
\text { quimioterapia } \\
\text { - Piora de quadro clínico } \\
\text { - Novo ciclo de } \\
\text { quimioterapia }\end{array}$ \\
\hline M11 & $\begin{array}{l}\text { - Ferida em vulva e dor ao urinar } \\
\text { - Ginecologista em julho, } 2013 \text { na } \\
\text { Unidade de um município vizinho } \\
\text { - Biópsia e retirada do tumor }\end{array}$ & $\begin{array}{l}\text { - Câncer de vulga } \\
\text { - Encaminhamento para } \\
\text { oncologista }\end{array}$ & $\begin{array}{l}\text { - Inicia radioterapia em } \\
\text { novembro, } 2014 \\
\text { - Consulta clínico geral } \\
\text { particular } \\
\text { - Novo ciclo de } \\
\text { quimioterapia }\end{array}$ \\
\hline M12 & $\begin{array}{l}\text { - Sangramento vaginal intenso } \\
\text { - Atendimento em Unidade Básica } \\
\text { em fevereiro, } 2015 \\
\text { - Encaminhamento para serviço de } \\
\text { emergência } \\
\text { - Encaminhamento para } \\
\text { ginecologista e biópsia }\end{array}$ & $\begin{array}{l}\text { - Câncer de útero } \\
\text { - Exames pré- } \\
\text { operatórios }\end{array}$ & $\begin{array}{l}\text { - Retorna à ginecologista e } \\
\text { cirurgia em março, } 2015 \\
\text { - Encaminhada para } \\
\text { oncologista } \\
\text { - Radioterapia em abril, } \\
2015 \\
\text { - Linfonodos aumentados } \\
\text { na região inguinal } \\
\text { - Encaminhada para } \\
\text { serviço de emergência por } \\
\text { suspeita de apendicite } \\
\text { - Internação hospitalar, } \\
\text { tomografia e biópsia em } \\
\text { setembro, } 2015 \\
\text { - Metástase em região } \\
\text { inguinal e coccígea } \\
\text { - Quimioterapia em } \\
\text { setembro, } 2015 \text { em } \\
\text { internação hospitalar }\end{array}$ \\
\hline
\end{tabular}


A busca das mulheres pelo diagnóstico e tratamento do câncer foi dotada de muitos caminhos. Em meio à peregrinação, dificuldades foram mencionadas para obter o diagnóstico de câncer como, falta de orientações pelos profissionais de saúde, burocracia e tempo demasiado na fila de espera por exames de média e alta complexidade pelo SUS ou consultas especializadas.

Em algumas situações, no primeiro contato da paciente com atendimento de saúde, o profissional suspeitou da gravidade da condição clínica da paciente e a encaminhou para serviço de emergência e, a partir dali já foram para internação hospitalar. Outras mulheres acessaram a rede de saúde em um modo misto, sendo mais rápido ao complementarem o atendimento do sistema publicado com o do sistema privado de saúde, assim como com a judicialização. Entretanto, em meios aos problemas de acesso aos serviços de saúde, foi citada a importância dos profissionais da Atenção Primária em Saúde para o diagnóstico precoce do câncer.

[...] no início eu tinha uma dor no peito, daí eu fui na ginecologista e ela falou que era problema de mama ou costela quebrada. Eu fiz exames e não deu nada, aí eu fui a tantos médicos que eu apaguei [...] em maio é que comecei a procurar os médicos, cada um dizia uma coisa, outro dizia outra [...] em setembro do ano passado 2014 saiu o diagnóstico quando eu estava hospitalizada [...]em 15 de junho de 2015 é que comecei a medicação da quimioterapia. [...] para conseguir o tratamento da quimioterapia procurei a justiça, só demorou porque a Dra. esqueceu de me dar uns papeis [...] dos primeiros sintomas, até a certeza do diagnóstico que a gente vai num médico, vai no outro e ninguém sabe de nada, é ruim, cada um fazia um exame, e não sabia o que era, esta incerteza que faz a gente peregrinar pelo sistema (M1).

Em 2011 eu fiz minha primeira mamografia, no resultado desta mamografia dizia em baixo que tinha que complementar com uma ultrassonografia, só que esta ultrassonografia saiu em 2013 [...] em maio/ junho de 2014 comecei a sentir um caroço em baixo

do seio [...] pronto a biópsia que concluiu que era câncer de mama que tinha passado para o pulmão, nisto tudo já estávamos em 2014 [...]fiz a cirurgia retirei todos os nódulos e, até hoje eu continuo fazendo

a quimioterapia [...]. Estou aguardando a outra quimioterapia pela justiça há 4 meses [...] enquanto isto eu estou fazendo esta, que eu deveria ter feito só 4 ciclos, já estou no $11^{\circ}$ ciclo, até chegar o Herceptim. [...] ninguém me avisou que tinha que fazer aquilo rápido [ultrassonografia]. Agora cura no sistema que a gente vive, não tem [...] tem que ser via juiz federal porque estadual não deu (M2).

O posto eu ia regularmente, todos os anos eu fazia pré-câncer, e fazia todos os exames que a médica solicitava. A partir dos 40 eu fazia regularmente todos os anos a mamografia [...]. Dia 10 de junho de 2015 eu fiz cirurgia, e comecei a quimioterapia dia 06 de agosto [...]tenho 49 anos hoje [...] em 2013 a médica do posto não quis me dar a mamografia, disse que era de 2 em 2 anos [...] depois em março de 2015 eu consegui a nova Dra. que entrou no posto [...] a médica me encaminhou pro mastologista, [...]até eu fazer a biópsia faltou 2 dias para completar 2 meses [...] para conseguir o tratamento da quimioterapia foi 1 mês depois da cirurgia [...] para conseguir o atendimento aqui eu tive que ir lá na secretaria de saúde pra eles me liberarem a quimioterapia [...] já vi que estava alterado, estava na categoria IV, em final de novembro em 2014 saiu o resultado [...] pediram tudo pelo SUS, eu paguei todos, porque tu sabe se a pessoa esperar pelo SUS [...] Dra. do posto faz uns 3 anos que me pediu uma endoscopia e eu até hoje estou esperando [...]tive que pagar os exames para ser mais rápido, o problema é esta secretaria de saúde, que para tu ver um ginecologista que é um médico corriqueiro, faz mais de 2 meses e não me chamaram (M3).

[...] e sempre por ficha fica difícil, tem que ir de madrugada [Unidade Básica de Saúde] e às vezes tem que agendar, aí não dá [...]. Eu comecei a emagrecer, desarranjo [...]quando deu nos intestinos, tive que

fazer 27 sessões de radioterapia, e 10 ciclos de quimioterapia para depois fazer a cirurgia [...]retirou parte do intestino e colocou a bolsinha. Na tomografia e mamografia e mostrou a lesão [...] estou em tratamento com a oncologista, agora fui encaminhada para o mastologista que ele que vai fazer a cirurgia também (M4).

[...] começou mesmo com uma cistite, não curava, depois foi aparecer sangue na urina [...] sentia dor [...] isto foi fim de julho, início de agosto foi descoberto mesmo, diagnosticado, mas os primeiros sintomas

foram em janeiro, fevereiro deste ano iniciou. Eu fiquei uns quantos meses sem procurar médico nada achando que era normal, [...] o primeiro médico que eu procurei foi um nefrologista, ele pediu um ultrassom, no outro dia já foi feito, o médico disse na hora que era um tumor, [...], voltei no nefrologista ele me deu um encaminhamento pro PS [Pronto Socorro], no do PS me encaminharam pra HE [Hospital Escola] 
fiquei internada por duas semanas, foi onde pediram tudo que foi exame, inclusive a biopsia, tomografia, eletro, exames de sangue fizeram tudo[...] e nisto me encaminharam pro oncologista, [...] fui até a secretaria de saúde pra liberar o tratamento (M5).

[...] com sangue na urina, depois botei hemorragia 2 dias, procurei o médico no posto [...] ele pediu uns exames, mas não achou nada, ele me encaminhou para urologista, em Piratini e me pediu uns exames, fiz a biópsia em Piratini, era câncer. A primeira quimioterapia

foi em 28 de agosto, faço de 21 em 21 dias, e a radioterapia será depois da quimioterapia [...] (M6).

[...] foi no banho, eu estava me apalpando e quando eu senti [...]. Fui no posto fiz o meu CP [Citopatológico],

falei para enfermeira que tinha sentido no banho dois carocinhos e ela me examino e já pediu a minha mamografia [...] ]eu descobri a doença em 2010, no posto [...]o clínico me encaminhou para o mastologista, ele fez a cirurgia, colocou a prótese, [...]. Em dezembro em 2010 fiz 25 sessões de radioterapia. Faço Aredia para os ossos,

uma vez por mês [...]. Tomo o Amaxafeno que eu levo daqui todos os meses, [...]. Depois da radioterapia que eu comecei a tomar, em setembro de 2011 (M7).

[...]passei a mão por um nódulo bem representativo

[...] eu vinha fazendo uns exames periódicos [...] na última mamografia que eu fiz, tinha um alerta que eu tinha que repetir em meio ano, eu não vi aquilo, mas também não estou culpando a Dra., mas ela também

não me falou [...] depois fui na secretaria de saúde para marcar um mastologista [...] consegui para o dia 18 de janeiro de lá no CERON [Centro de Radioterapia

e Oncologia]. Fiz a biópsia e a cirurgia, isto tudo

foi particular [...] eu fiz a cirurgia em 25 de janeiro de 2010. [...] consegui marcar uma consulta com a oncologista que é daqui pelo SUS (HE) com quem eu me trato até hoje. [...] fiz as sessões de quimioterapia,

16 sessões, todo o ano de 2010 foi de tratamento, eu sei que em setembro eu terminei a quimioterapia e em dezembro eu terminei a radioterapia. Eu fiz 35 sessões de radioterapia, porque eu fiz nas duas mamas [...] continuei consultando até que em 2013

senti uma dor no peito, e descobri uma metástase no esterno [...] trocou o quimioterápico oral, mas no final de 2014 me apareceu outra metástase no $5^{\circ}$ arco

costal e já estava com 2 metástases, [...] ela trocou novamente o medicamento, não adiantou de nada. Em um dos exames fiz uma ultrassonografia e descobri as metástases no fígado, passei para quimioterapia EV de novo desde final junho deste ano (M8).
[...] eu tinha muita dor no estômago, fui ao clinico geral pela Unimed [convênio médico] e ele me solicitou uma endoscopia, quando saiu o resultado deu adenocarcinoma grau II, fui encaminhada para um cirurgião [...] este tratamento que estou fazendo é o segundo porque deu recidiva da doença, eu procurei o cirurgião, fiz a cirurgia e retirei $75 \%$ do estômago,

e depois da cirurgia fui encaminhada para um oncologista, o oncologista me deu o tratamento da quimioterapia, fiz oito sessões, da primeira vez que me deu comecei em junho do ano passado 2014 e terminei em dezembro, e neste período eu fiquei só no acompanhamento oncológico, neste meio tempo

eu perdi o plano de saúde, [...] fui na prefeitura do meu município e pedi o encaminhamento para o oncologista, eles me encaminharam aqui para HE [...] a primeira tomografia daqui demorou um certo tempo para fazer [...] quando veio o resultado eu já estava

com um nódulo no abdome [...] A médica daqui a oncologista me encaminhou pro cirurgião, fiz cirurgia, dessa vez eu fiz paga a cirurgia para ser mais rápida, [...] foi para biópsia e deu adenocarcinoma de novo, era recidiva mesmo, [...] passei pra oncologista e ela me passou a quimioterapia de novo [...] iniciei esta quimioterapia dia 17 de setembro são 12 ciclos o total, é no mínimo 6 meses o tratamento (M9).

[...] foi através do exame de rotina mesmo que o médico pediu [...] vim de um encaminhamento lá do posto, para oncologista me avaliar [...] eu baixei o hospital e já fiz a biópsia [....]fiz 36 sessões de radioterapia, e quimioterapia eu fiz uma semana no início da radioterapia, e uma semana no fim [...]perdi a minha filha mais velha com 25 anos, então aí o impacto né, ele deu uma crescida, a oncologista disse para fazer 6 ciclos desse medicamento [...] (M10).

[...]desconfiei que tivesse uma ferida na vulva, e parecia um fungo, [...] quando eu me dei conta, já estava avançado, enraizado, [...]com muita dor para urinar não aguentava mais [...] a Dra. do posto me encaminhou para fazer a cirurgia, colhe o material dali para ver o que era [biópsia]. [...]tirei o tumor, [...] passou uns oito meses, apareceu na virilha, um nódulo, fui para a cirurgia para retirar o tumor. De janeiro de 2015 para cá e que apareceu, agora está nos ossos. E é só a quimioterapia que eu faço mesmo [...] Sou de Canguçu, e os primeiros médicos foram de lá, eu consultei com a ginecologista de lá [...] quando saiu o resultado, as minhas filhas começaram a correr atrás de médico, iam num, iam noutro [...]. A burocracia é grande e os encaminhamentos errados. Ninguém se interessa em te dizer: tu tens que ir por aquele caminho ali, que é o caminho certo. Isto aí que é o problema. 
Mandaram primeiro procurar o Hospital privado, lá foi nós, não era para o médico de lá. Isto particular foi. Depois lá eles disseram tu tens que procurar um cirurgião. Ninguém te indica, [...] falando com uma pessoa e outra, nos indicaram a Dra. do CERON [Centro de Radioterapia e Oncologia]. [...] foi onde conseguimos consultar (M11).

[...] fui no postinho e ela me mandou para o PS [Pronto Socorro] e do PS para um hospital, a médica a ginecologista me mandou para um ambulatório e mandou para a biópsia. E quando deu o resultado disse que era tumor maligno [...]. Eu fui lá, fiz os exames pré-operatórios, uns eu paguei, os de sangue e de urina, que iam demorar mais, e o eletrocardiograma eu fiz lá.

Em 16 de março eu operei, eles mandaram para fazer radioterapia, fiz 25 sessões um mês após a cirurgia foi em abril, [...] a médica tinha pedido a tomografia fazia quatro meses e não tinham me chamado ainda, a médica falou: tu estás liberada, tu estás curada, não tens mais câncer. Dra. faz uns quatro dias que estou sentindo uma bolinha aqui na minha virilha. Me mandou para o OS, fui para o hospital e fiquei 19 dias internada, fiz a tal da tomografia [...] viram que o tumor voltou bem na virilha, se espalhando pelas costas já [...] (M12).

\section{Discussão}

Pelo relato da $\mathrm{M} 2$, o tempo transcorrido entre a primeira mamografia (2011), até a realização da ultrassonografia (2013) para comprovação da alteração do exame anterior e a consulta com a ginecologista para o encaminhamento ao mastologista foi de três anos, sendo dois anos somente pela espera de um exame: a ultrassonografia. No caso da M4 novamente o impasse se dá pela dificuldade no acesso aos exames, em que um ano transcorreu entre a realização da coIonoscopia, a biópsia e descobrir o tumor e ser encaminhada para o serviço de oncologia.

Há de se pensar em maior agilidade na realização dos exames dos usuários do sistema de saúde, sendo este o principal recurso para o diagnóstico de doenças, como o câncer, que requer tempo hábil para o início do tratamento, pois a detecção precoce está intimamente ligada à cura de tal enfermidade. Estudo realizado com 600 mulheres com câncer de mama no Distrito Federal identificou que $28,9 \%$ do itinerário foi iniciado na atenção primária com atendimento subsequente no serviço de tratamento. O maior tempo entre a primeira consulta e o tratamento foi de muIheres com menor renda familiar, realização da primeira consulta em serviços públicos ${ }^{11}$.

Os depoimentos das mulheres indicam que as mesmas têm dificuldade para identificar sinais e sintomas que exijam procurar atendimento de saúde. Até que a mulher sinta de fato algo concreto que a faça procurar atendimento na rede de saúde, a doença já está evoluindo e, possivelmente avançando de estadiamento. Estudo de revisão identificou que a demora na busca por diagnóstico desde a manifestação dos primeiros sinais e sintomas do adoecimento, pode ser influenciada por processos socioculturais aprendidos no decorrer da vida e provenientes do seio familiar, tais como uso de chás, assim como em decorrência de insucessos obtidos no sistema de saúde formal ${ }^{12}$.

A oferta e disponibilidade de exames de média e alta complexidade e consultas especializadas em tempo hábil para obter diagnóstico o mais precoce possível não é realidade no SUS. Diante disso, os usuários recorrem, inclusive por recomendação dos próprios profissionais da rede, a um fluxo alternativo entre o sistema público e privado, em que o tempo entre a realização e a liberação do laudo é incomparavelmente mais rápido neste último.

Estudo que buscou conhecer o tempo percorrido pela usuária com suspeita de câncer de mama e até o seu diagnóstico, foi de 120 a 180 dias de espera para a maioria das mulheres ${ }^{13}$. Revisão integrativa analisou a rede de cuidado ao câncer de mama desde a primeira consulta até seu diagnóstico e identificou entre as dificuldades a espera prolongada da usuária na obtenção de seu diagnóstico e consequências graves por conta desta demora ${ }^{14}$. Os estudos supracitados, bem como esta pesquisa, indicam que é necessária a conexão entre os serviços do SUS, buscando efetividade nas linhas de cuidado oncológico, além de aceleração à assistência integral e resolutiva às mulheres.

No caminho percorrido por M3, ela expressa que a médica poderia ter solicitado mamografia um ano antes. Os protocolos assistenciais, como os do Ministério da Saúde para rastreamento de câncer de mama, são fundamentais para guiar a prática dos profissionais de saúde e padronizar condutas, mas há situações que se devem considerar os fatores de risco, a queixa da usuária e juntamente a identificação de achados clínicos, tendo um fluxo de avaliação diferenciado. 
Apenas pelo relato da usuária não é possível saber se a tomada de decisão da profissional foi correta, ou não, contudo se chama atenção para a relevância do conhecimento dos protocolos e fluxos de encaminhamento, embora complexos, são adaptáveis a cada caso e determinam como se dará o acesso aos tratamentos na rede SUS, buscando-se agilidade, de acordo com as necessidades dos usuários. O Ministério da Saúde recomenda que a periodicidade do rastreamento com mamografia nas faixas etárias recomendadas de 50 a 69 anos seja a bienal ${ }^{15}$.

Tanto no relato de M3, como de M8 foi mencionado a necessidade de ir até à secretaria de saúde para a liberação da quimioterapia, sendo este relatado pela primeira e a segunda procurou o serviço para consultar com um especialista. Esse trajeto percorrido demonstra o fluxo das usuárias que irão realizar o procedimento ambulatorial mediante a requisição médica, juntamente com um formulário de Autorização de Procedimentos de Alta Complexidade, que é por meio deste que os ambulatórios e hospitais recebem os recursos financeiros para o tratamento oncológico, conforme prevê a Portaria 296 de julho de $1999^{16}$.

Nesse sentido de regulação dos serviços de saúde, cabe ressaltar que o fluxo percorrido por M10 não é o correto, perante o preconizado, ainda que tenha sido o mais resolutivo, o profissional da unidade básica, na qual foi atendida, não deveria ter feito a referência da usuária direta para o hospital e sim a encaminhado para oncologista, ou se necessário para o Pronto Socorro, para que o mesmo avaliasse a usuária e tomasse as próximas condutas, como a referência para um hospital para então proceder com seu trajeto na rede via SUS, conforme prevê a Portaria $n^{\circ} 1.559$ de agosto de 2008, que institui a Política Nacional de Regulação do SUS, distribuindo os serviços em saúde com maior equidade ${ }^{17}$.

Diante disso, faz-se necessário mencionar possíveis percalços no sistema de saúde, não somente no atraso dos exames diagnósticos, como no olhar clínico do profissional de saúde para os prováveis achados no exame físico em uma consulta de rotina. Como se aponta na declaração da usuária M3 em que ela diz sentir um caroço no seio e a profissional da saúde não solicita a mamografia para afastar a suspeita de câncer de mama, porque está fora da rotina habitual de solicitação do exame. Ou até mesmo quando a M12 em uma consulta após o tratamento oncológico detecta uma saliência na região inguinal e é encami- nhada para o Pronto Socorro por possível diagnóstico de "apendicite". Ou ainda quando no resultado da mamografia da M2 solicita a confirmação com uma ultrassonografia.

Também é identificado a ausência de advertência ao profissional quanto a possível seriedade da situação em virtude da demora do sistema de modo que poderia ser solicitado uma maior agilidade na realização do exame, ou ainda a usuária ter a prioridade por se tratar de suspeita de câncer. No relato da M8, ela referiu que tinha no resultado de uma mamografia um alerta de necessidade de repetição do exame de mamografia em seis meses, por se tratar de uma suspeita de câncer. Embora a usuária não culpabilize o profissional de saúde por não ter lhe avisado sobre a repetição do exame, houve um descaso na prevenção, o que deixou a participante do estudo tranquila com o resultado da mamografia, já que a rotina é bienal.

Contudo, é importante destacar o papel da enfermeira no relato da $\mathrm{M7}$, em que a mesma acolheu a usuária do sistema único de saúde, dando a devida atenção ao achado clínico, procedendo com a realização do exame físico, a coleta do citopatológico e solicitando a mamografia, mostrando o quanto a atuação deste profissional de saúde, aumenta a capacidade de rastreamento da população alvo, intervindo na prevenção do câncer e seus agravos. A enfermagem desempenha papel importante na promoção da saúde e a prevenção das patologias. No que se refere ao câncer de colo de útero a profissão exerce a propagação de informações para a prevenção, a consulta de enfermagem e a efetuação do exame citopatológico, colaborando para o rastreamento deste tipo câncer ${ }^{18}$.

Entretanto ainda há lacunas no que se refere ao entendimento de profissionais da saúde na detecção da doença. Estudo que buscou identificar o conhecimento e a utilização de métodos para detecção do câncer de útero por profissionais da saúde, entendeu que os profissionais conhecem e realizam os métodos de detecção, porém sem sempre apresentam comportamento eficaz na sua utilização, o que expõe as mulheres a um risco ainda maior de desenvolver este tipo de câncer ${ }^{19}$. Este comportamento por vezes ineficaz por parte dos profissionais impacta diretamente no prognóstico positivo nos casos de identificação da doença, devido à má utilização do tempo. O tempo de sobrevida na oncologia é utilizado de acordo com os métodos disponíveis de rastreamento e necessita ser considerado. 
O rastreamento do câncer permite amplitude do tempo de sobrevida tendo em vista o tempo de antecipação, de duração e do sobre diagnóstico. O câncer de mama que é o que mais prevalece nas mulheres brasileiras por exemplo, quando diagnosticado precocemente tem chances de uma sobrevida de $97 \%$ em cinco anos ${ }^{20}$.

A demora pela busca do atendimento médico por parte das usuárias do serviço de saúde, dificulta o rastreamento e por consequência o diagnóstico precoce, diminuindo o tempo de sobrevida, problema que é possível identificar a partir dos relatos da M5 e M11, o quanto a demora pela procura prejudicou as participantes do estudo, por contribuir para o avanço da doença. Por intermédio da explanação da M11 foi possível se deparar com a ideia principal que surgiu neste estudo, ou seja, a importância de conhecer os fluxos e os serviços de referência no setor da oncologia. Para onde e quando referenciar?

Diante do impacto de saber da doença e muitas vezes por desconhecer o caminho a percorrer, as participantes do estudo recorrem ao setor privado, ora por despesas com consultas médicas, ora por exames diagnósticos, como foi possível identificar no relato de várias participantes. Com a biópsia em mãos é possível à liberação da consulta com o oncologista pela secretaria municipal de saúde, por meio do encaminhamento médico, para a liberação do tratamento adequado, conforme prevê a Portaria $n^{\circ} 874$ de maio de 201321, quando institui a Política Nacional de Atenção Oncológica, garantindo o acesso aos usuários nas esferas federativas, em cada nível de atenção, segundo o planejamento dos princípios e diretrizes do SUS, a universalidade, equidade, regionalização, hierarquização e integralidade da atenção à saúde.

Para que a trajetória percorrida para o tratamento do câncer possa garantir os princípios e diretrizes do SUS, faz-se necessária a organização dos serviços de saúde em todos os níveis de atenção. A pactuação entre os serviços fortalece a linha de cuidado e permite agilidade ao acesso à assistência e tratamento ${ }^{14}$.

Outra questão levantada é a prioridade de atendimento e realização de exames de média e alta complexidade para usuários que tenham doenças com agravos como o câncer, ainda que a demanda seja alta, inclusive para esta parcela da população, devido ao aumento de número de casos anualmente ${ }^{22}$, há de se pensar em maior agilidade para a realização dos procedimentos e exames de alta complexidade, conforme prevê a Portaria $n^{\circ} 741 / 2005^{23}$, que define os serviços, estabelece prazos, fluxos e exigências mínimas para integrar a rede de atenção oncológica.

A equidade, princípio do sistema público de saúde brasileiro, também está envolvida no estudo, uma vez que as usuárias que possuíam recursos financeiros necessários para a realização dos exames conseguiam maior agilidade em seu tratamento quando comparadas as usuárias dependentes do SUS. Há gargalos, como as longas filas pela espera para a realização de um exame, todavia o subfinanciamento do setor público de saúde implica na oferta de exames ser menor que a demanda da população. Entretanto, a avaliação do funcionamento da rede, conhecimento das trajetórias das usuárias nesta rede e investimento nas informações para os profissionais de saúde parece ser questões que não exigem uma considerável aplicação financeira para que possam ser realizadas. Os enfermeiros, que além de serem profissionais bem atuantes na rede de atenção básica de saúde, estão igualmente presentes, trabalhando em espaços de gestão, como as secretarias municipais de saúde, podendo contribuir com seus saberes para que os fluxos aconteçam da maneira mais adequada.

\section{Considerações finais}

Os fluxos de usuárias do SUS não obedecem a uma linha retilínea, como prevê as linhas de cuidado em oncologia, partindo da atenção primária para os níveis de maior complexidade. Em alguns casos as usuárias já diretamente buscaram os especialistas, com o intuito de não esperar longos prazos pela consulta no SUS, optando por arcar com as despesas médicas e com exames. Desta forma, percebeu-se a fragilidade no acesso às consultas especializadas e aos exames de média e alta complexidade pelo SUS, o que impacta negativamente na assistência integral aos usuários.

Identificou-se que a demora no fluxo de acesso das usuárias, parte dos primeiros sintomas ao diagnóstico, sendo considerado rápido o acesso ao tratamento após o diagnóstico de câncer, por já ser uma garantia legal. Ressalta-se que o reconhecimento dos primeiros sintomas por uma profissional de saúde pode demorar, quando a pessoa não procure tão logo serviços de saúde para verificar se trata de algum problema importante. 
Barreiras no acesso à atenção básica igualmente foi relatada pelas participantes deste estudo, tais como: dificuldade para conseguir o atendimento médico, sendo necessário chegar de madrugada para conseguir uma ficha, a falta de profissionais nas unidades de saúde, alta rotatividade, o que interfere na construção do vínculo e corresponsabilização pelo usuário e falta de orientações ou encaminhamentos que não atendem as necessidades de saúde. Cabe destacar que neste estudo foi dispendioso analisar os depoimentos e, também durante a coleta, ao se associar as datas, lembrar os primeiros sintomas, quando estes surgiram e quando exatamente foi a busca pelo atendimento. A entrevista com 12 mulheres foi suficiente para contemplar os objetivos do estudo.

Entretanto, a realização da pesquisa, em apenas um município, pode ser um limite de generalização do estudo, ao abarcar algumas características particulares regionais da rede de saúde, que possa não representar a qualidade dos fluxos de atenção em saúde das pessoas com câncer de outras localidades do Brasil. Outro limite de generalização do estudo, consiste no tipo de coleta de dados da investigação que se deu a partir das narrativas das mulheres sobre sua trajetória até o acesso ao tratamento quimioterápico, devido ao caráter subjetivo e autobiográfico que não garantem exatidão em relação ao tempo cronológico e as condutas dos profissionais de saúde, de modo que não se possa validar a narrativa das usuárias, que estabelecem julgamento sobre condutas ou tratamentos inadequados.

Dessa maneira, a pesquisa ao trazer o conhecimento dos múltiplos caminhos que as pessoas percorrem para a atenção em saúde em tempo hábil, igualmente suscita reflexões que podem contribuir com a construção de estratégias para aperfeiçoar os fluxos de encaminhamento na rede de saúde do município do sul do Brasil e em outros municípios, pois conforme a literatura, a realidade não é tão diferente em outros locais. Especificamente, esse estudo, fornece elementos para auxiliar na elaboração de um fluxo de atenção às pessoas com câncer na rede de saúde do município estudado, podendo ser aplicável também a outras localidades. Um ponto frágil, que parece ser essencial, trata-se da disseminação de informações da gestão municipal e da busca dos profissionais por apropriarem-se da oferta de serviços e como acontece esse acesso na rede de saúde e que possam utilizar as melhores estratégias de orientação da população.

\section{Contribuições das autoras}

Neujahr TS participou da concepção, delineamento, coleta e análise dos dados da pesquisa, interpretação dos resultados, redação do artigo científico. Porto AR participou da concepção, delineamento, análise e interpretação dos resultados, redação do artigo científico. Gonçalves KD participou da análise e interpretação dos resultados e redação do artigo científico. Thofehrn MB, Hammes HR e Geppert AK participaram da análise e interpretação dos resultados e redação do artigo científico.

\section{Conflitos de interesses}

Nenhum conflito financeiro, legal ou político envolvendo terceiros (governo, empresas e fundações privadas, etc.) foi declarado para nenhum aspecto do trabalho submetido (incluindo, mas não se limitando a subvenções e financiamentos, participação em conselho consultivo, desenho de estudo, preparação de manuscrito, análise estatística, etc.).

\section{Referências}

1. Vidotto PCP, Ferrari RAP, Tacla MTGM, Facio BC. Experiência materna no itinerário diagnóstico do câncer infantil. Rev Enferm UFPE. 2017;11(4):1565-73. doi: 10.5205/reuol.9763-85423-1SM.1104201702

2. Ministério da Saúde. Instituto Nacional do Câncer. ABC do câncer, Abordagens Básicas para o controle do câncer. [Internet]. 2011. Disponível em: http://bvsms.saude.gov.br/bvs/publicacoes/ inca/abc_do_cancer_2ed.pdf>

3. Teston EF, Fukumori EFC, Benedetti GMS, Spigolon DN, Costa MAR, Marcon SS. Sentimentos e dificuldades vivenciadas por pacientes oncológicos ao longo dos itinerários diagnóstico e terapêutico. Esc Anna Nery. 2018;22(4):e20180017. doi: 10.1590/2177-9465-ean-2018-0017

4. Souza KA, Souza SR, Tocantins FR, Freitas TF, Pacheco PQC. O itinerário terapêutico do paciente em tratamento oncológico: implicações para a prática de enfermagem. Ciênc Cuid Saúde. 2016;15(2):259-67. doi: 10.4025/cienccuidsaude.v15i2.29896

5. Sarmento LS, Silva LF, Goes FGB, Paiva ED, Depianti JRB. A visão dos familiares quanto às orientações realizadas junto à criança em quimioterapia antineoplásica. Cogitare Enferm. 2016;21(1):1-9. doi: $\underline{10.15446 / a v . e n f e r m . v 34 n 1.37390}$

6. Feijó AM, Linck CL, Viegas AC, Santos BP. Os caminhos de cuidado das mulheres com diagnóstico de câncer de mama. Av Enferm. 2016;34(1):58-8. doi: 10.15446/av.enferm.v34n1.37390

7. Leite JF, Dimenstein M, Paiva R, Carvalho L, Amorim AKMA, França $A$. Sentidos da saúde numa perspectiva de gênero: um estudo com homens da cidade de Natal/RN. Psicologia: Ciência e Profissão. 2016;36(2):341-53. doi: 10.1590/1982-3703001812013 
8. Ministerio da Saude. Departamento de Análise de Saúde e Vigilância de Doenças não Transmissíveis. Sistema de Informação sobre Mortalidade. [Internet]. 2019. Disponivel em: http://svs.aids. gov.br/dantps/

9. Oliveira JM, Reis JB, Silva RA. Busca por cuidado oncológico: percepção de pacientes e familiares. Rev Enferm UFPE online. 2018;12(4):938-46. doi: 10.5205/1981-8963-v12i4a23135 9p938-946-2018

10. Minayo MCS, Deslandes SF, Cruz Neto O, Gomes R. Pesquisa social: teoria, método e criatividade. Rio de Janeiro: Vozes; 2013.

11. Barros AF, Araújo JM, Murta-Nascimento C, Dias A. Itinerário terapêutico de mulheres com câncer de mama tratadas no Distrito Federal, Brasil. Rev Saúde Pública. 2019;53:14. doi: 10.11606/s1518-8787.2019053000406

12. Soares DA, Santos EM, Arruda IS. Itinerários terapêuticos de pessoas com câncer: produção científica no Brasil. Rev APS. 2017;20(1):118-29. doi: 10.34019/1809-8363.2017.v20.15906

13 Traldi MC, Galvão P, Morais SS, Fonseca MRCC. Demora no diagnóstico de câncer de mama de mulheres atendidas no Sistema Público de Saúde. Cad Saúde Colet. 2016;24(2):185-91. doi: 10.1590/1414-462X201600020026

14. Aguiar FAR, Sousa TC, Branco JGO, Costa FBC, Torres ARA, Arruda LP. Produção do cuidado na rede de atenção ao câncer de mama: revisão integrativa. Sanare. 2018;17(1):84-92.

15. Ministério da Saúde. Protocolos da Atenção Básica: Saúde das Mulheres. [Internet]. 2016. Disponível em: http://bvsms. saude.gov.br/bvs/publicacoes/protocolos_atencao_basica_saude_ mulheres.pdf

16. Brasil. Ministério da Saúde. Portaria SAS/MS n 296/99 - dispõe sobre quimioterápicos. Procede sobre a normalização técnica e operacional relativa ao Sistema de Autorização de Procedimentos Ambulatoriais de Alta Complexidade/Custo APAC. Ministério da saúde. Secretaria de Assistência à Saúde, 1999.

17. Brasil. Ministério da Saúde. Portaria $n^{\circ} 1.559$, de $1^{\circ}$ de agosto de 2008. Institui a Política de Nacional de Regulação do Sistema Único de saúde (SUS). Ministério da Saúde. Gabinete do Ministro, 2008.

18. Aoyama EA, Pimentel AS, Andrade JS, Daniel WV, Souza RAG, Lemos LR. Assistência de enfermagem na prevenção do câncer de colo do útero. Brazilian Journal of Health Review. 2018;2(1):162-70.

19. Almeida AF, Holmes ES, Lacerda CCC, Farias CF, Costa MBS, Santos SR. Métodos de detecção de câncer de colo uterino entre profissionais da saúde. Rev Enferm UFPE online. 2015;9(1):62-8. doi: 10.5205/reuol.6817-60679-1-ED.0901201509
20. Migowski A. A detecção precoce do câncer de mama e a interpretação dos resultados de estudos de sobrevida. Ciênc Saúde Coletiva. 2015;20(4):1309. doi: 10.1590/1413$\underline{81232015204.17772014}$

21. Brasil. Ministério da Saúde. Portaria $n^{\circ} 874$, de 16 de maio de 2013. Institui a Política Nacional para a Prevenção e Controle do Câncer na Rede de Atenção à Saúde das Pessoas com Doenças Crônicas no âmbito do Sistema Único de Saúde (SUS). Ministério da Saúde. Gabinete do Ministro, 2013.

22. Brasil. Instituto Nacional de Câncer José Alencar Gomes da Silva. Coordenação de Prevenção e Vigilância. Estimativa 2018: incidência de câncer no Brasil. [Internet]. 2017. Disponível em: http://www1.inca.gov.br/estimativa/2018/estimativa-2018.pdf

23. Brasil. Ministério da Saúde. Portaria $n^{\circ} 741$, de 19 de dezembro de 2005. Dispõe sobre a classificação dos Serviços de saúde em oncologia. Ministério da Saúde, 2005. 